First publ. in: Naturwissenschaften 84 (1997), 9, pp. 389-397

\section{Molecular Phylogenetic Information on the Identity of the Closest Living Rela- tive(s) of Land Vertebrates}

\author{
Rafael Zardoya ${ }^{1}$ and Axel Meyer ${ }^{2}$ \\ Department of Ecology and Evolution \\ and Program in Genetics, State University \\ of New York, Stony Brook, \\ New York 11794-5245, USA
}

The phylogenetic position of tetrapods relative to the other two living sarcopterygian lineages (lungfishes and the coelacanth) has been subject to debate for many decades, yet remains unresolved. There are three possible alternatives for the phylogenetic relationships among these three living lineages of sarcopterygians, i.e., lungfish as living sister group of tetrapods, the coelacanth as closest living relative of tetrapods, and lungfish and coelacanth equally closely related to tetrapods. To resolve this important evolutionary question several molecular data sets have been collected in recent years, the largest being the almost complete 28S rRNA gene sequences (about $3500 \mathrm{bp}$ ) and the complete mitochondrial genomes of the coelacanth and a lungfish (about $16500 \mathrm{bp}$ each). Phylogenetic analyses of several molecular data sets had not provided unequivocal support for any of the three hypotheses. However, a lungfish+tetrapod or a lungfish+coelacanth clade were predominantly favored over a coelacanth+tetrapod grouping when the entire mitochondrial genomes alone or in combination with the nuclear 28S rRNA gene data were analyzed with maximum parsimony, neighbor-joining, and maximum likelihood phylogenetic methods. Also, current paleontological and morphological data seem to concur with these molecular results. Therefore the currently available molecular data seems to rule out a coelacanth+tetrapod relationship, the traditional textbook hypothesis. These tentative molecular phylogenetic results point to the inherent difficulty in resolving relationships among lineages which apparently originated in rapid succession during the Devonian.
T he transition from life in water to life on land was perhaps one of the most significant events in the evolution of vertebrates (e.g. Panchen and Smithson 1987). This major evolutionary transition involved many sweeping morphological (e.g., evolution of limbs and air breathing), physiological, and behavioral changes, but nonetheless it seems to have taken place within an astonishingly short period of time of only 10-20 million years (Carroll 1988; Maisey 1996; Cloutier and Ahlberg 1996). It is well established that the early tetrapods evolved from lobe-finned fishes (Sarcopterygii) and not from rayfinned fishes (Actinopterygii; e.g., Romer 1966). However, due to the general scarcity of fossils, and the difficulty in character definitions, hence homology assignments, it has been highly controversial which lineage of the sarcopterygians is the one most closely related to land vertebrates (Cloutier and Ahlberg 1996; Forey 1988; Schultze 1994; for a review see Meyer 1995). Sarcopterygians are currently divided into three major groups (Table 1): Rhipidistia, Actinistia, and Dipnoi (e.g., Carroll 1988; Schultze 1994; Table 1). Rhipidistians are comprised out of at least four extinct lineages (Porolepiformes, Osteolepiformes, Rhizodontida, and Elpistostegalia) and the extant Tetrapoda (Table 1; e.g., Carroll 1988; Schultze 1994; but see, e.g., Ahlberg 1991; Cloutier and Ahlberg 1996; Janvier 1996, who include dipnoans within Rhipidistia).

Recent paleontological evidence strongly suggests that elpistostegids (or panderichthyids) are the sister group of tetrapods (Vorobyeva and Schultze 1991; Ahlberg et al. 1996), and that these obligatory aqua-

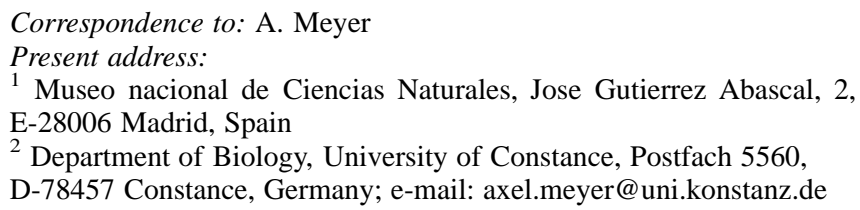

${ }^{1}$ Museo nacional de Ciencias Naturales, Jose Gutierrez Abascal, 2, E-28006 Madrid, Spain

2 Department of Biology, University of Constance, Postfach 5560,

D-78457 Constance, Germany; e-mail: axel.meyer@uni.konstanz.de 
Table 1. Classification of fish (modified from Carroll 1988; Cloutier and Ahlberg 1996; Schultze 1994; for many authors, e.g., Janvier 1996; Cloutier and Ahlberg 1996, the dipnoans should be included within the Rhipidistia ${ }^{\dagger}$ extinct)

Class: Chondrichthyes (cartilaginous fish)

Class: Osteichthyes (bony fish)

Subclass: Actinopterygii (ray-finned fish)

Chondrostei (sturgeon, Acipenser; bichir, Polypterus)

Neopterygii (gar, Lepisosteus; bowfish, Amia; modern ray-finned fish, Teleostei)

Subclass: Sarcopterygii (lobe-finned fish) (Romer 1955)

Actinistia (coelacanth, Latimeria) (Cope 1871)

Dipnoi (lungfish, Neoceratodus, Protopterus, Lepidosiren) (Muller 1885)

Rhipidistia (Cope 1887)

Porolepiformes (Jarvik 1942)

Rhizodontid (Andrews and Westoll 1970)

Osteolepiformes (Berg 1937)

Elpistostegalia (Camp and Allison 1961)

Tetrapoda (land vertebrates)

Lissamphibia (modern amphibians)

Amniota (reptiles, birds, mammals)

tic fishes already possessed many of the morphological preadaptations and innovations that are required for life on land. Actinistia or hollow-spinned fishes were once a highly successful group of lobe-finned fishes from the Early Devonian to the Late Cretaceous. Due to their absence from the fossil record after the Late Cretaceous (70-80 mya), these fishes were thought to have gone extinct until the sensational discovery of the only surviving species, the coelacanth (Latimeria chalumnae) from the Comoro Islands in the Indian Ocean (Smith 1939, 1956; Schliewen et al. 1993). Dipnoi, the lungfishes, are a highly modified group of air-breathing lobe-finned fishes, which were abundant and diversified in the Late Devonian. However, now they are reduced to only three relict genera that are found in Australia, Africa, and South America (Neoceratodus, Protopterus, and Lepidosiren; Forey 1987; Cloutier and Forey 1991). Despite much research there is still no general agreement regarding the phylogenetic relationships among Actinistia, Dipnoi, and Rhipidistia (including Tetrapoda; Ahlberg 1991; Cloutier and Ahlberg 1996; Forey 1988, 1991; Long 1995; Maisey 1996; Marshall and Schultze 1992; Meyer 1995; Nelson 1994; Panchen and Smithson 1987; Rosen et al. 1981; Schultze 1994). This controversy will continue until new relevant fossils of intermediate forms connecting the three groups are discovered, and agreement among paleontologists about the homology of some characters (e.g., the choanae) is achieved (Forey 1988; Schultze 1994; Rosen et al. 1981).

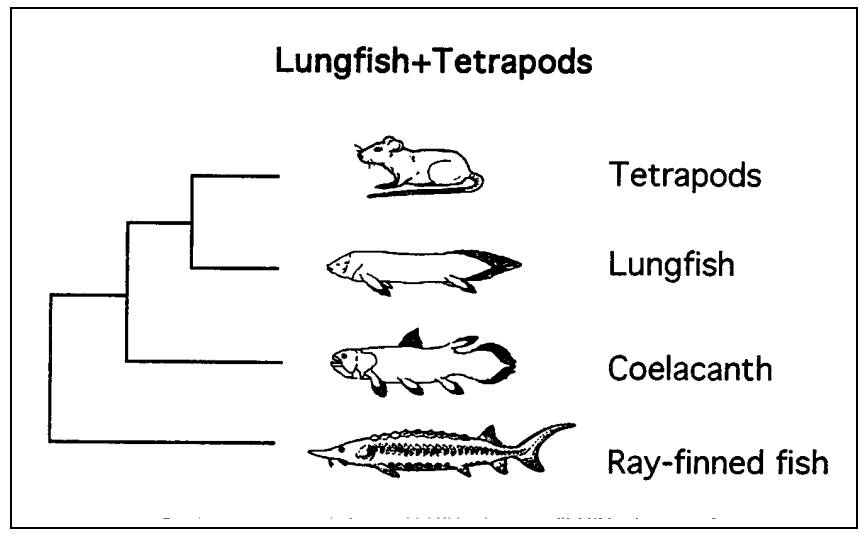

Fig. 1. Lungfishes as closest living sister group of tetrapods. This hypothesis is preferred by many paleontologists and morphologists. It is also supported by several mitochondrial protein-coding genes (see text)

An approach based on molecular rather than on morphological data from lungfishes, the coelacanth, and tetrapods, the only living representatives of sarcopterygians, might be helpful to solve this controversy (reviewed by Meyer 1995). Knowledge of the phylogenetic relationships among the three living lineages of lobe-finned fishes should be instrumental when assigning homologies and determining the direction of character transformation in the transition from life in water to life on land. Three possible alternative hypotheses can account for the phylogenetic relationships among extant lineages of sarcopterygians: (a) the lungfishes are the closest living relative of tetrapods (Fig. 1), (b) the coelacanth is the living sister group of tetrapods (Fig. 2), and (c) both lungfishes and the coelacanth are equally closely related to tetrapods (Fig. 3). Until recently most studies on the living sarcopterygians did not include both the coelacanth and the lungfishes because their primary goal was not to elucidate their phylogenetic relationships (e.g., Hillis et al. 1991; Kolb et al. 1974). Therefore due to the incomplete sampling of species these studies could not discriminate among any of the three competing hypotheses (reviewed in Meyer 1995).

During the past ten years, however, mitochondrial and nuclear sequences have been collected from both the coelacanth and the lungfishes with the explicit goal of resolving this phylogenetic issue (e.g., Gorr et al. 1991; Hedges et al. 1993; Meyer and Dolven 1992; Meyer and Wilson 1990; Fig. 4). Even more recently the almost complete $28 \mathrm{~S}$ rRNA gene (Zardoya and Meyer 1996c) and the entire mitochondrial genomes of these two taxa (Zardoya and Meyer 1996a, 1997; Zardoya et al. 1997) have been determined and make up the currently largest molecular data set collected so far to address this evolutionary 


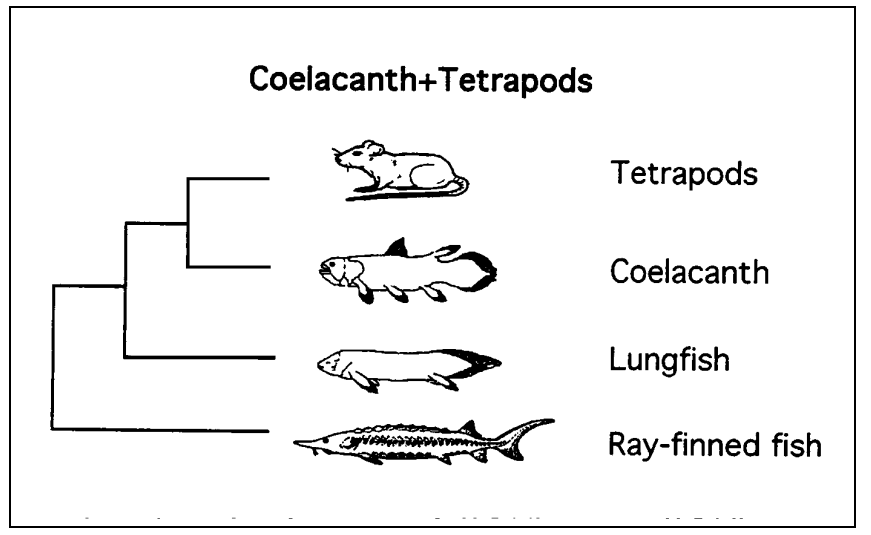

Fig. 2. The coelacanth as closest living relative of tetrapods. Ever since the sensational discovery of living specimens of the coelacanth, this hypothesis has been favored in most general biology textbooks. However, morphological and molecular data provide only weak support for it

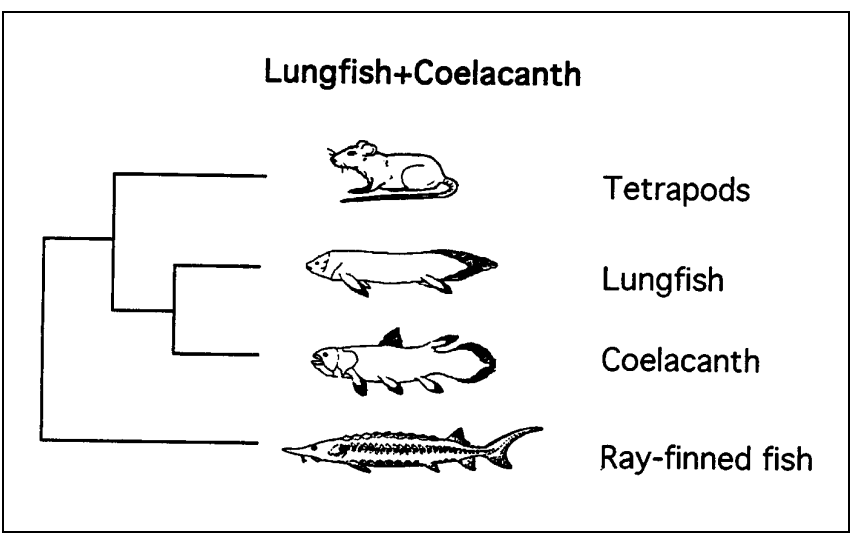

Fig. 3. Lungfishes and the coelacanth are equally closely related to tetrapods. Traditionally this hypothesis has received less attention than the alternatives. However, recent morphological and molecular phylogenetic analyses (nuclear 28S rRNA genes, mitochondrial tRNA genes, and several mitochondrial protein-coding genes) support the lungfish+coelacanth grouping

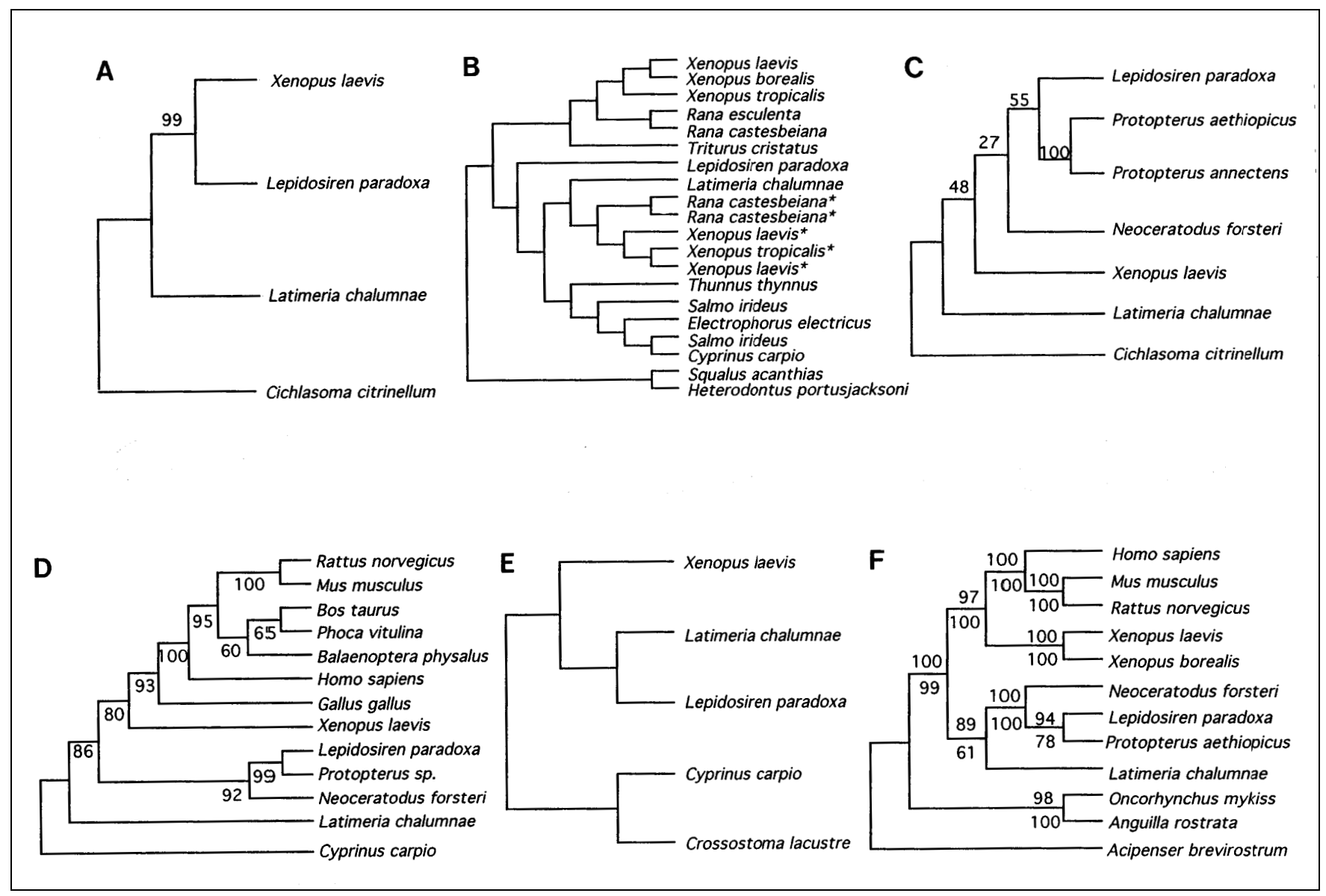

Fig. 4. Living sarcopterygian phylogenetic relationships based on different molecular data sets. A) Maximum parsimony analysis of mitochondrial 12S rRNA and Cyt $b$ gene fragments (Meyer and Wilson 1990). B) UPGMA analysis based on $\beta$ hemoglobin sequences (Gorr et al. 1991). C) Maximum parsimony analysis of a mitochondrial $12 \mathrm{~S}$ rRNA gene fragment (Meyer and Dolven 1992). D) Neighbor-joining analysis of the complete mitochondrial $12 \mathrm{~S}$ and 16S rRNA genes (Hedges et al. 1993). E) Maximum likelihood analysis of the complete mitochondrial COI gene (at the amino acid level; Yokobori et al. 1994). F) Maximum parsimony, neighbor-joining, and maximum likelihood analyses of the almost complete nuclear 28S rRNA gene (Zardoya and Meyer 1996c) 


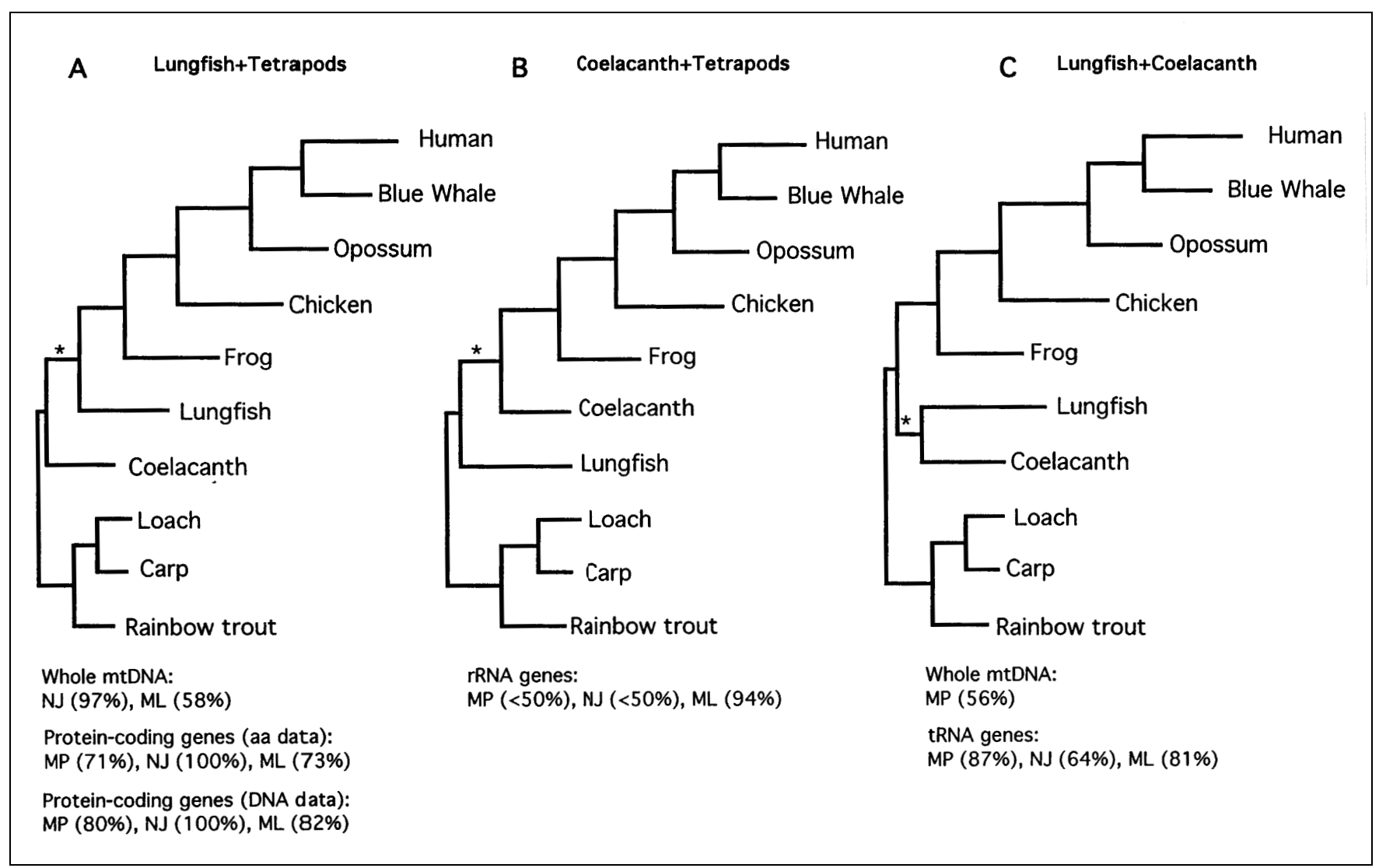

Fig. 5. Mitochondrial DNA phylogenetic analyses of the phylogenetic relationships of the living sarcopterygian lineages. A) MP, NJ, and ML analyses of the mitochondrial protein-coding gene data set (both at the amino acid and at the DNA level), and NJ and ML analyses of the whole mitochondrial data set supported the hypothesis of lungfish as the closest living sister group of tetrapods. B) Coelacanth as closest living relative of tetrapods was weakly favored by the mitochondrial

problem. The goal of these studies is to establish the phylogenetic relationships among the three extant lineages of sarcopterygians through the analysis of molecular data sets. However, a well-supported answer to this question might aid in recreating the sequence of evolutionary events that permitted sarcopterygian fishes to colonize land.

\section{Lungfishes as Closest Living Sister Group of Tetrapods}

The first DNA data set that supported the hypothesis that lungfishes are the closest living relatives of tetrapods (Fig. 1) was collected by Meyer and Wilson (1990). A total of $664 \mathrm{bp}$ from two fragments of the mitochondrial $12 \mathrm{~S}$ rRNA and cytochrome $b$ genes were sequenced from a ray-finned fish (Cichlasoma
rRNA gene data set regardless of the phylogenetic method of inference. C) A lungfish+coelacanth clade is supported by the whole mitochondrial data set when analyzed with MP, and by the mitochondrial tRNA data set with MP, NJ, and ML phylogenetic methods of inference. Bootstrap values for the nodes (asterisks) are shown for each of the phylogenetic analyses

citrinellum), the coelacanth (Latimeria chalumnae), and the South American lungfish (Lepidosiren paradoxa) and compared with those of a tetrapod (Xenopus laevis). Maximum parsimony analyses of this data set, excluding third codon positions of the cytochrome $b$ gene, and using the ray-finned fish as outgroup, arrived at a single most parsimonious tree in which the lungfish+tetrapod clade was supported by a $99 \%$ bootstrap value in 100 replicates (Fig. 4). In bootstrapping Felsenstein (1985) replicates (e.g., 100-1000) of the original data set are generated by random sampling with replacement. These multiple data sets are subjected to phylogenetic analyses, and a (typically 50\%-majority rule bootstrap) consensus of the multiple generated trees is calculated. For each node of the consensus tree a bootstrap value is assigned based on the frequency of its occurrence in the multiple bootstrap trees. The higher the bootstrap values are (close to $100 \%$ ) the more confidence is 
Table 2. Statistical support for the three competing hypotheses on the relationships among living sarcopterygians lineages

\begin{tabular}{|c|c|c|c|c|c|c|c|c|c|c|c|c|c|c|}
\hline \multirow[t]{2}{*}{ Tree (DNA level) } & \multicolumn{2}{|c|}{ ATPase 6} & \multicolumn{2}{|c|}{ ATPase 8} & \multicolumn{2}{|l|}{$\mathrm{COI}$} & \multicolumn{2}{|c|}{ COII } & \multicolumn{2}{|l|}{ COIII } & \multicolumn{4}{|l|}{ Cyt $b$} \\
\hline & $\Delta$ & SE & $\Delta$ & SE & $\Delta$ & SE & $\Delta$ & SE & $\Delta$ & $\mathrm{SE}$ & $\Delta$ & SE & & \\
\hline Coelacanth+tetrapods & -4.7 & \pm 4.2 & \multicolumn{2}{|c|}{ ML (516.4) } & \multicolumn{2}{|c|}{$-9.1 \quad \pm 8.6$} & \multicolumn{2}{|c|}{ ML (2105.3) } & \multicolumn{2}{|c|}{$-7.8 \quad \pm 6.0$} & \multicolumn{2}{|c|}{$\begin{array}{l}\text { ML (3823.8) } \\
-11.5 \quad \pm 10.2\end{array}$} & & \\
\hline Lungfish+coelacanth & \multicolumn{2}{|c|}{ ML (2705.4) } & -1.1 & \pm 1.5 & \multirow{2}{*}{\multicolumn{2}{|c|}{$\begin{array}{l}\text { ML (3462.2) } \\
1004\end{array}$}} & -1 & \pm 5.9 & ML & $186.0)$ & -18.9 & \pm 8.0 & & \\
\hline Length & \multicolumn{2}{|c|}{362} & \multicolumn{2}{|l|}{68} & & & \multicolumn{2}{|c|}{428} & \multicolumn{2}{|c|}{516} & \multicolumn{4}{|l|}{752} \\
\hline & $\Delta$ & SE & $\Delta$ & SE & $\Delta$ & $\mathrm{SE}$ & $\Delta$ & SE & $\Delta$ & $\mathrm{SE}$ & $\Delta$ & SE & $\Delta$ & SE \\
\hline Lungfish+tetrapods & \multicolumn{2}{|c|}{ ML (3519.7) } & \multicolumn{2}{|c|}{ ML (4774.2) } & \multicolumn{2}{|c|}{ ML (1306.1) } & \multicolumn{2}{|c|}{ ML (5595.9) } & \multicolumn{2}{|c|}{ ML (1390.5) } & \multirow{3}{*}{\multicolumn{2}{|c|}{$\begin{array}{lr}-4.6 & \pm 8.7 \\
-11 & \pm 7.0 \\
\text { ML }(5850.6) \\
980\end{array}$}} & ML & 582.8) \\
\hline Coelacanth+tetrapods & -14 & \pm 8.7 & -6.5 & \pm 5.2 & -1.8 & \pm 2.4 & -5 . & \pm 6.0 & -3.5 & \pm 3.0 & & & -1.2 & \pm 2.3 \\
\hline Lungfish+coelacanth & -15 & \pm 8.4 & -4.8 & \pm 5.8 & -0.1 & \pm 3.9 & -6 & \pm 5.6 & -3.4 & \pm 3.2 & & & -0.3 & \pm 2.9 \\
\hline Lungfish, Tetrapods & ML & $8220.0)$ & -17 & \pm 10 & -13 & \pm 7.8 & -16 & \pm 9.9 & -7.9 & \pm 22.2 & & & & \\
\hline Coelacanth, Tetrapods & -46 & \pm 20.9 & ML (1 & $13468.9)$ & -8.3 & \pm 9.1 & -15 & \pm 10.2 & -31 & \pm 25 & & & & \\
\hline Lungfish, Coelacanth & -20 & \pm 16.5 & -18 & \pm 9.8 & ML $(9$ & 347.6) & ML & $11505.5)$ & ML & $2580.0)$ & & & & \\
\hline Length & 6858 & & 2101 & & 1417 & & 322 & & 1359 & & & & & \\
\hline
\end{tabular}

The log-likelihood of the ML tree in given in parentheses, and the differences in log-likelihood of alternative trees from that of the ML tree ( $\Delta$ ) are shown with their SE (following \pm ), which were estimated by the formula of Kishino and Hasegawa (1989). Third codon positions of protein coding genes were excluded from the analyses at the DNA level

placed into the reality of a given node based on the collected data.

Normark et al. (1991) extended the cytochrome $b$ analysis by the addition of a second African lungfish (Protopterus sp.) and several more ray-finned fish DNA sequences. These data continued to favor a lungfish+tetrapod grouping albeit with low bootstrap support (51\%). However, when the African (Protopterus aethiopicus and Protopterus annectens) and the Australian (Neoceratodus forsteri) lungfishes were included into an extended study of the mitochondrial 12S rRNA gene (Meyer and Dolven 1992), an unresolved coelacanth+lungfish+tetrapod trichotomy (the lungfish+tetrapod clade was favored in the most parsimonious tree but with low bootstrap support of only $48 \%$ ) resulted (Fig. 4). Several studies have recently pointed out the poor performance of individual mitochondrial genes (or fragments of them) in recovering the precise phylogenetic relationships of vertebrate taxa at a deep-branch level and consequently the need of analyzing larger data sets (Cummings et al. 1995; Russo et al. 1996; Zardoya and Meyer 1996b).

Hedges et al. (1993) claimed to have found stronger support for the lungfish+tetrapod clade based on an analysis of the complete $12 \mathrm{~S}$ and $16 \mathrm{~S}$ rRNA mito- chondrial genes (Fig. 4). This data set was comprised in total of $2903 \mathrm{bp}$ from the three lungfish genera, the coelacanth, eight tetrapods, and one ray-finned fish (as an outgroup taxon). Neighbor-joining analyses of this data set based on Kimura corrected distances resulted in a lungfish+tetrapod clade, which was supported by a relatively high bootstrap value (86\%; Fig. 4). Moreover, a maximum parsimony analysis also favored lungfish as the closest living relative of tetrapods, but unfortunately no bootstrap values were provided in the original paper to assess the support of this node (Hedges et al. 1993). A reanalysis of this data set with all three commonly used methods of phylogenetic inference (maximum likelihood, ML; maximum parsimony, MP, and neighbor-joining, $\mathrm{NJ}$ ) resulted in an unresolved lungfish+coelacanth+tetrapod trichotomy (also see below; Zardoya and Meyer 1996c, Zardoya and Meyer 1997; Zardoya et al. 1997).

Analyses of a very large data set of all 13 proteincoding genes encoded by the mitochondrial genome (11736 characters) with all three commonly used phylogenetic methods of phylogenetic inference (MP, NJ, and ML) provided support for the lungfish+ tetrapod grouping (Zardoya and Meyer 1996c, 1997; Zardoya et al. 1997; Fig. 5A). The lungfish+tetrapod 
node was supported by an $80 \%$ bootstrap value in the MP analysis when third codon positions and transitions in first codon positions were excluded (Fig. 5A). Moreover, similar results were obtained when transitions in first codon positions were included in the analysis, or different weights for transitions and transversions were assumed for any position. The bootstrap support for the lungfish+tetrapod node in the NJ analysis (using Kimura distances and correcting for rate variation among sites with a $\gamma$ distribution $a$ shape parameter) was $100 \%$ (Fig. 5A). In the ML analysis (using empirical base frequencies and transition/transversion ratios with the HKY85 model) (Hasegawa et al. 1985), all branch lengths were found to be significantly greater than zero $(P<0.01)$, and the bootstrap value for the lungfish+tetrapod node was $82 \%$ (Fig. 5A). Statistical confidence of the best trees of the MP and ML analyses was moreover evaluated using the formula of Kishino and Hasegawa (1989) and Templeton (1983; Table 2). These methods calculate the standard error of the difference in log-likelihood or number of steps between the best tree and its competing hypotheses. According to these tests, the protein-coding genes, although providing overall support for a lungfish+ tetrapod clade, could not statistically reject a lungfish+coelacanth clade. However, the relationship of the coelacanth as closest sister group of tetrapods was clearly rejected (Table 2). Interestingly, the lungfish+tetrapod clade was supported by several NADH dehydrogenase genes and cytochrome $b$ but not by the cytochrome oxidase or ATPase genes when a Kishino-Hasegawa test was performed (Zardoya et al. 1997; Table 2). Similar results were obtained when the analysis was performed based on inferred amino acids (3887 characters) rather than nucleotides. Furthermore, when this data set was combined with the rest of the mitochondrially encoded (rRNA and tRNA) genes, it also supported lungfishes as closest living sister group of tetrapods with MP (except when third codon positions and transitions in first codon positions were excluded), NJ, and ML (Zardoya et al. 1997).

\section{The Coelacanth as Closest Living Sister Group of Tetrapods}

Although the idea that the coelacanth is the "missing link" between fishes and tetrapods is still prevalent in many general biology texts (e.g., Kämpfe et al. 1980; Romer 1966), the hypothesis of the coelacanth as closest living sister group of tetrapods (Fig. 2) re- ceived the least support in all phylogenetic analyses of molecular data (Meyer 1995). This hypothesis was erroneously favored by Gorr et al. (1991) based on their analysis of the amino acid sequences of $\alpha$ and $\beta$ hemoglobin chains (Fig. 4). The UPGMA method was used to analyze these amino acid sequences, taken from two sharks, several ray-finned fishes, the South American lungfish, the coelacanth, and several amphibians (adults and tadpoles). According to their results, the $\beta$ chains of the coelacanth were most similar to those of larval amphibians, and this was interpreted as support for a coelacanth+tetrapod relationship (Fig. 4). However, serious flaws have been identified in this study (Meyer and Wilson 1991; Sharp et al. 1991; Stock and Swofford 1991). For instance, the unequal rates of evolution of the different lineages under study violate the assumptions of the UPGMA method, and more importantly, the assumption of orthology between the fish and amphibian hemoglobin sequences was not proven. Reanalyses of the data with MP (Meyer and Wilson 1991; Sharp et al. 1991; Stock and Swofford 1991) and ML (Yokobori et al. 1994) showed that the $\beta$-hemoglobin data tended weakly to support the coelacanth+tetrapod clade. However, a reanalysis of the $a$-chain with ML slightly favored a lungfish+coelacanth clade (Yokobori et al. 1994).

Similarly, phylogenetic analyses of the almost complete nuclear 18S rRNA gene sequences (about 1800 nucleotides) with MP arrived at a single most parsimonious tree in which the coelacanth+tetrapod clade was preferred (Stock et al. 1991). However, this topology contained many unorthodox groupings weakly supported in the bootstrap analysis, and therefore it is generally accepted that the 18S rRNA gene is not appropriate for addressing this phylogenetic question (Meyer 1995).

A coelacanth+tetrapod grouping is also suggested by the analysis of the lipid and myelin protein content of the nerve membranes in vertebrate taxa (Tamai et al. 1994; Waehneldt et al. 1991; and references therein). In particular, the detection of low levels of $2^{\prime}, 3^{\prime}$ cyclic nucleotide $3^{\prime}$-phosphohydrolase activity, a myelin marker enzyme, and the higher occurrence of proteolipid protein in the coelacanth but not in lungfishes was interpreted as support for a close relationship between the coelacanth and tetrapods since high levels of $2^{\prime}, 3^{\prime}$-cyclic nucleotide $3^{\prime}$-phosphohydrolase activity and proteolipid protein are found in amphibians and mammals but not in ray-finned fishes (Tamai et al. 1994; Waehneldt et al. 1991). However, the phylogenetic validity of these results needs to be interpreted with caution because the presence of similar lipid and myelin protein contents provides only weak information on common descent or, the alterna- 
tive, evolutionary convergence (Meyer 1995; Ax 1988).

With the sequencing of the African lungfish (Protopterus dolloi) and the coelacanth complete mitochondrial genomes (Zardoya and Meyer 1996a, 1997), a phylogenetic reanalysis of the previously published mitochondrial $12 \mathrm{~S}$ and $16 \mathrm{~S}$ rRNA gene sequences became possible (Zardoya et al. 1997). The $3^{\prime}$ end (last $332 \mathrm{bp}$ ) of the 16S rRNA gene of the coelacanth sequence of this new data set differed from those of Hedges et al. (1993). This fragment is likely a contamination with alligator $16 \mathrm{~S}$ rRNA sequence, which was also determined in that laboratory (Hedges 1994). Interestingly, a coelacanth+tetrapod clade was preferred rather than the lungfish+tetrapod clade when the new uncontaminated rRNA gene sequences (2101 characters) were analyzed with MP, NJ (using Kimura distances and correcting for rate variation among sites with a gamma distribution $a$ shape parameter), and ML (using empirical base frequencies and transition/transversion ratios with the HKY85 model) using teleosts (rainbow trout, carp, and loach) as outgroup taxa (Fig. 5B). However, the coelacanth+tetrapod node had a bootstrap value below $50 \%$ both in the MP and the NJ analyses (interestingly, this node was favored by a $94 \%$ bootstrap support in the ML analysis; Fig. 5B). This apparent lack of resolution of the rRNA data set was confirmed with the Kishino-Hasegawa and the Templeton statistical tests, which could not rule out the two alternative hypotheses (Table 2; Zardoya et al. 1997), suggesting that mitochondrial $12 \mathrm{~S}$ and $16 \mathrm{~S}$ rRNA genes are inappropriate phylogenetic markers for this question.

\section{The Coelacanth and the Lungfishes Are Equally Closely Related to Tetrapods}

The alternative that lungfishes are most closely related to the coelacanth, and therefore, that a combined lungfish+coelacanth clade constitutes the closest living sister group of tetrapods (Fig. 3) was initially not widely considered, but support (from morphological and molecular data) in its favor has accumulated recently (Chang 1991; Schultze 1994; Yokobori et al. 1994; Zardoya and Meyer 1996c).

The first molecular phylogenetic support for this hypothesis came from the phylogenetic analyses of the mitochondrial cytochrome oxidase I (COI) gene (Yokobori et al. 1994). The amino acid sequences deduced from the COI gene nucleotide sequences (about $1550 \mathrm{bp}$ ), of the South American lungfish, and the coelacanth were subjected to ML, MP, and NJ analyses using the carp and loach as outgroup taxa and the frog as single representative of the tetrapods (Fig. 4). All phylogenetic analyses of this gene strongly supported (with bootstrap values above $90 \%$ ) a lungfish+coelacanth clade. Nevertheless, a less strong result was obtained when a Kishino-Hasegawa test of the combination of the COI data set with the earlier 12S rRNA and cytochrome $b$ data sets was performed. A coelacanth+tetrapod clade was statistically ruled out, but equal support was found for a lungfish+tetrapod clade and a lungfish+coelacanth clade (Yokobori et al. 1994).

The lungfish+coelacanth clade was also the most preferred of the three alternative hypotheses when COII, COIII, ATPase 6, ATPase 8, ND5, and ND6 genes were analyzed at the amino acid level using ML, and teleosts (rainbow trout, carp, and loach) as outgroup taxa (Zardoya et al. 1997). Similar results were obtained at the DNA level when third codon position were excluded from the analyses (Table 2). However, in none of the cases could either of the two alternative hypotheses, i.e., lungfishes or the coelacanth as closest living sister group of tetrapods, be statistically ruled out (Table 2 ).

The analysis of a data set combining the 22 mitochondrial tRNA gene sequences (1604 characters) with MP, NJ (using Kimura distances and correcting for rate variation among sites with a $\gamma$ distribution $a$ shape parameter), and ML (using empirical base frequencies and transition/transversion ratios with the HKY85 model), using teleosts (rainbow trout, carp, and loach) as outgroup taxa, also favored a lungfish+coelacanth clade (Zardoya et al. 1997; Fig. 5C). The lungfish+coelacanth node was supported by $87 \%, 64 \%$, and $81 \%$ bootstrap values in the MP, NJ, and ML analyses, respectively (Fig. 5C). Nonetheless, according to the Kishino-Hasegawa and Templeton tests, the two alternative hypotheses could not be rejected with this data set (Table 2).

All mitochondrial genes combined favored the lungfish+coelacanth clade when third codon positions and transitions in first codon positions of proteincoding genes were excluded from the MP analysis. The MP bootstrap support for the lungfish+coelacanth node was only 56\% (Zardoya et al. 1997; Fig. 5C).

Finally, the lungfish+coelacanth hypothesis also was favored when the nuclear 28S rRNA sequences from the coelacanth, three lungfish genera, human, mouse, rat, Kenyan clawed frog, clawed frog, rainbow trout, and eel were analyzed with all three common methods of phylogenetic inference (MP, NJ, ML) using the sturgeon (Acipenser brevirostrum) as outgroup (Zardoya and Meyer 1996c; Fig. 4). The data set 
consisted of 4786 sites, and a transition:transversion ratio of 2:1 was used in the phylogenetic analyses. The robustness of the results was confirmed by the moderately high bootstrap values obtained for the lungfish+coelacanth node in the NJ (61\%) and MP $(89 \%)$ trees. However, neither of the two alternative hypotheses had significantly lower log-likelihoods than the favored maximum likelihood tree when the Kishino-Hasegawa test was performed (Zardoya and Meyer 1996c; Table 2).

\section{Molecular Evidence on the Living Sister Group of Tetrapods Using All Available Data}

The phylogenetic analyses of the combined mitochondrial and nuclear data sets (20926 characters) with all three commonly used methods of phylogenetic inference were not entirely conclusive. For the MP analysis (a) third codon positions of mitochondrial protein coding genes were excluded, (b) a transition:transversion weight of 1:2 was applied for the 28S rRNA data set, and (c) human and frog were used as representatives of tetrapods, and the rainbow trout was used as outgroup. The MP analysis of the combined nuclear and mitochondrial data set arrived at a single most parsimonious tree in which a lungfish+coelacanth clade was weakly favored with a bootstrap support below 50\% (Zardoya et al. 1997). Under the same conditions the NJ analysis, however, supported a lungfish+tetrapod clade with a bootstrap value of $90 \%$. Finally, ML analyses supported a lungfish+coelacanth relationship when the 10-taxon mitochondrial data set and the 12-taxon 28S rRNA data set (Zardoya and Meyer 1996c) were combined. In this case a $79 \%$ or a $61 \%$ bootstrap support were obtained for the lungfish+coelacanth node, when the mitochondrial protein-coding gene subset was analyzed at the amino acid level or at the DNA level, respectively (Zardoya et al. 1997). However, if a Kishino-Hasegawa test was performed, neither of the two alternative hypotheses could be statistically ruled out with this data set (Zardoya et al. 1997; Table 2).

In conclusion, the overall evidence seems to support both the lungfish+coelacanth (Fig. 1) and a lungfish+ tetrapod (Fig. 3) hypotheses, but not the coelacanth+ tetrapod grouping (Fig. 2). The lungfish+ coelacanth clade was supported by the nuclear $28 \mathrm{~S}$ rRNA gene data (Zardoya and Meyer 1996c), and the mitochondrial ATPase 6, ATPase 8, COI, COII, COIII, ND5, and tRNA data (Zardoya et al. 1997). The lungfish+tetrapod clade was strongly supported by the mitochondrial ND1, ND2, ND3, ND4L, ND4, ND6, and cyt $b$ genes (and all mitochondrial protein coding genes combined). Therefore it appears that the more slowly evolving genes (cytochrome oxidase subunits, tRNAs and 28S rRNA) favor the lungfish+coelacanth hypothesis, whereas relatively more rapidly evolving genes (e.g., NADH subunits and cytochrome $b$ ) support the lungfish+tetrapod grouping. Although it cannot be statistically rejected in all cases, the coelacanth+tetrapod hypothesis seems to be the most unlikely of the three. These results are in agreement with most recent morphological evidence which supports mainly the lungfish+coelacanth and the lungfish+tetrapod hypotheses over the coelacanth+tetrapod hypothesis (Ahlberg 1991; Chang 1991; Cloutier and Ahlberg 1996; Forey 1987, 1988; Forey et al. 1991; Janvier 1996; Long 1995; Panchen and Smithson 1987; Schultze 1994). The rapid origin of sarcopterygian lineages within a narrow window in time (20 million years, dating back to the Devonian) seems to be mainly responsible for the tentative evidence so far obtained by Meyer (1995). Future studies on this question will require the collection of nuclear protein coding gene sequence data. From a mitochondrial point of view, the sequencing of new amphibian mitochondrial genomes will also be desirable because the available Xenopus laevis sequence (Roe et al. 1985) is likely to contain sequencing errors and additional urodele and caecilian sequences (Zardoya and Meyer, in preparation) will divide the long branch leading to the single amphibian complete mitochondrial DNA sequence available. It is well known that long branches in phylogenetic trees attract each other leading to wrong phylogenetic inferences (Felsenstein 1978). Despite many potential difficulties, future work using a combined data approach that utilizes molecular and morphological data should also be attempted. The resolution of the phylogenetic relationships among living sarcopterygian lineages via molecular studies should provide a framework that would help in discerning among proposed alternative hypotheses Ax (1988) that also include extinct lineages of lobe-finned fishes. A molecular framework might contribute to a better understanding of the relationships among the extinct lineages of rhipidistians that led to the origin of tetrapods and will aid in the interpretation and polarization of the morphological and physiological traits of sarcopterygians that predate the colonization of land.

Acknowledgements. R.Z. was sponsored by a postdoctoral grant of the Ministerio de Educacion y Ciencia of Spain. This work received partial financial support from grants from the National Science Foundation (BSR-9107838, BSR-9119867, DEB-9615178) and from a colla- 
boration grant with the Max-Planck-Institut für Biologie in Tübingen, Germany to A.M.

Ahlberg PE (1991) A re-examination of sarcopterygian interrelationships, with special reference to the porolepiformes. Zool J Linnean Soc 103:241-288

Ahlberg PE, Clack JA, Luksevics E (1996) Rapid braincase evolution between Panderichthys and the earliest tetrapods. Nature 381:61-63

Andrews SM, Westoll TS (1970) The postcranial skeleton of rhipidistian fishes excluding Eusthenopteron. Trans R Soc Edinburgh 68:391-489

Ax P (1988) Systematik in der Biologie. Fischer, Stuttgart

Berg LS (1937) A classification of fish-like vertebrates. Bull Acad Sci URSS, $1277-1280$

Camp CL, Allison HJ (1961) Bibliography of fossil vertebrates 1949_ 1953. Mem Geol Soc Am 84:1-53

Carroll RL (1988) Vertebrate paleontology and evolution. Freeman, New York

Chang MM (1991) Rhipidistians. In: Schultze HP, Trueb L (eds) Origins of the higher groups of tetrapods. Controversy and consensus. Cornell University Press, Ithaca, pp 3-28

Cloutier R, Ahlberg PE (1996) Interrelationships of basal sarcopterygians. In: Stiassny MLJ, Parenti LR, Johnson GD (eds) Interrelationships of fishes. Academic, San Diego, pp 445-479

Cloutier R, Forey PL (1991) Diversity of extinct and living actinistian fishes (Sarcopterygii). Env Biol Fish 32:59-74

Cope ED (1871) Contribution to the ichthyology of the Lesser Antilles. Trans Am Phil Soc 14:445-483

Cope ED (1887) Geology and Paleontology. Vertebrata. Am Nat 21:10141019

Cummings MP, Otto SP, Wakeley J (1995) Sampling properties of DNA sequence data in phylogenetic analysis. Mol Biol Evol 12:814-822

Felsenstein J (1978) The number of evolutionary trees. Syst Zool 27:2733

Felsenstein J (1985) Confidence limits on phylogenies: An approach using the bootstrap. Evolution 39:783-791

Forey PL (1987) Relationships of lungfishes. J Morphol [Suppl] 1:75-91

Forey PL (1988) Golden jubilee for the coelacanth Latimeria chalumnae. Nature 336:727-732

Forey PL, Gardiner BG, Patterson C (1991) The lungfish, the coelacanth and the cow revisited. In: Schultze HP, Trueb L (eds) Origins of the higher groups of tetrapods. Controversy and consensus. Cornell University Press, Ithaca, pp 145-174

Gorr T, Kleinschmidt T, Fricke H (1991) Close tetrapod relationships of the coelacanth Latimeria indicated by haemoglobin sequences. Nature 351:394-397

Hasegawa M, Kishino H, Yano T (1985) Dating of the human-ape splitting by a molecular clock of mitochondrial DNA. J Mol Evol 22:160174

Hedges SB (1994) Molecular evidence for the origin of birds. Proc Natl Acad Sci USA 91:2621-2624

Hedges SB, Hass CA, Maxson LR (1993) Relations of fish and tetrapods. Nature 363:501-502

Hillis DM, Dixon MT, Ammerman LK (1991) The relationships of the coelacanth Latimeria chalumnae: evidence from sequences of vertebrate 28S ribosomal RNA genes. Env Biol Fish 32:119-130

Janvier P (1996) Early vertebrates, vol 33. Oxford University Press, Oxford

Jarvik E (1942) On the structure of the snout of crossopterygians and lower gnathostomes in general. Zool Bidr Uppsala 21:235-675

Kämpfe L, Kittel R, Klapperstück J (1980) Leitfaden der Anatomie der Wirbeltiere. Fischer, Stuttgart

Kishino H, Hasegawa M (1989) Evaluation of the maximum likelihood estimate of the evolutionary tree topologies from DNA sequence data and the branching order in Hommoidea. J Mol Evol 29:170-179

Kolb E, Harris JI, Bridgen J (1974) Triose phosphate isomerase EC5.3.1.1 from the coelacanth: an approach to the rapid determination of an amino-acid sequence with small amounts of material. Biochem J 137:185-197

Long JA (1995) The rise of fishes: 500 million years of evolution. John Hopkins University Press, Baltimore London, p 220

Maisey JG (1996) Discovering fossil fishes. Holt, New York

Marshall C, Schultze HP (1992) Relative importance of molecular, neontological, and paleontological data in understanding the biology of the vertebrate invasion of land. J Mol Evol 35:93-101

Meyer A (1995) Molecular evidence on the origin of tetrapods and the relationships of the coelacanth. Trends Ecol Evol 10:111-116
Meyer A, Dolven SI (1992) Molecules, fossils and the origin of tetrapods. J Mol Evol 35:102-113

Meyer A, Wilson AC (1990) Origin of tetrapods inferred from their mitochondrial DNA affiliation to lungfish. J Mol Evol 31:359-364

Meyer A, Wilson AC (1991) Coelacanth's relationships. Nature 353:219

Müller J (1844) Über den Bau und die Grenzen der Ganoiden, und über das natürliche System der Fische. Ber Akad Wiss, Berlin 1844:67-85

Nelson GJ (1994) Fishes of the World, 3rd edn. Wiley, New York

Normark BB, McCune AR, Harrison RG (1991) Phylogenetic relationships of neopterygian fishes, inferred from mitochondrial DNA sequences. Mol Biol Evol 8:819-834

Panchen AL, Smithson TR (1987) Character diagnosis, fossils and the origin of tetrapods. Biol Rev 62:341-438

Roe BA, Din-Pow M, Wilson RK, Wong JF (1985) The complete nucleotide sequence of the Xenopus laevis mitochondrial genome. J Biol Chem 260:9759-9774

Romer AS (1955) Herpetichthyes, Amphibioidei, Choanichthys or Sarcopterygii. Nature 176:126

Romer AS (1966) Vertebrate paleontology, 3rd edn. University of Chicago Press, Chicago

Rosen DE, Forey PL, Gardiner BG, Patterson C (1981) Lungfishes, tetrapods, paleontology, and plesiomorphy. Bull Am Natl Mus Nat His 167:159-276

Russo CAM, Takezaki N, Nei M (1996) Efficiencies of different genes and different tree-building methods in recovering a known vertebrate phylogeny. Mol Biol Evol 13:525-536

Schliewen U, Fricke H, Schartl M, Epplen JT, Pääbo S (1993) Which home for the coelacanth? Nature 363:406

Schultze HP (1994) Comparison of hypotheses on the relationships of sarcopterygians. Syst Biol 43:155-173

Sharp PM, Lloyd AT, Higgins DG (1991) Coelacanth's relationships. Nature 353:218-219

Smith JLB (1939) A living fish of mesozoic type. Nature 143:455-456

Smith JLB (1956) Old fourlegs: the story of the coelacanth. Longmans, London, p 260

Stock DW, Swofford DL (1991) Coelacanth's relationships. Nature 353:217-218

Stock DW, Moberg KD, Maxson LR, Whitt GS (1991) A phylogenetic analysis of the $18 \mathrm{~S}$ ribosomal RNA sequence of coelacanth Latimeria chalumnae. Env Biol Fish 32:99-117

Tamai Y, Kojima H, Takayama-Abe K (1994) Lipids and myelin proteins in the brains of coelacanth (Latimeria chalumnae), lungfish (Lepidosiren paradoxa and Protopterus aetiopicus), bichir (Polypterus senegalus), and sturgeon (Acipenser ruthenus) (Osteichthyes): Phylogenetic implications. Can J Fish Aquat Sci 51:1265-1272

Templeton AR (1983) Phylogenetic inference from restriction endonuclease cleavage site maps with particular reference to the evolution of human and the apes. Evolution 37:221-244

Vorobyeva E, Schultze HP (1991) Description and systematics of panderichthyid fishes with comments on their relationship to tetrapods. In: Schultze HP, Trueb L (eds) Origins of the major groups of tetrapods: controversies and consensus. Cornell University Press, Ithaca, pp 68109

Waehneldt TV, Malotka J, Jeserich G, Matthieu JM (1991) Central nervous system myelin proteins of the coelacanth Latimeria chalumnae: phylogenetic implications. Env Biol Fish 32:131-143

Yokobori AI, Hasegawa M, Ueda T, Okada N, Nishikawa K, Watanabe K (1994) Relationship among coelacanths, lungfishes, and tetrapods: A phylogenetic analysis based on mitochondrial cytochrome oxidase I gene sequences. J Mol Evol 38:602-609

Zardoya R, Cao Y, Hasegawa M, Meyer A (1997) Searching for the closest Living relative(s) of tetrapods through evolutionary analyses of mitochondrial and nuclear data. Mol Biol Evol (submitted)

Zardoya R, Meyer A (1996a) The complete nucleotide sequence of the mitochondrial genome of the lungfish (Protopterus dolloi) supports its phylogenetic position as a close relative of land vertebrates. Genetics 142:1249-1263

Zardoya R, Meyer A (1996b) Evolutionary relationships of the coelacanth, lungfishes, and tetrapods based on the 28S ribosomal RNA gene. Proc Natl Acad Sci USA 93:5449-5454

Zardoya R, Meyer A (1996c) Phylogenetic performance of mitochondrial protein-coding genes in resolving relationships among vertebrates. Mol Biol Evol 13:933-942

Zardoya R, Meyer A (1997) The complete DNA sequence of the mitochondrial genome of a "living fossil", the coelacanth (Latimeria chalumnae). Genetics 146:995-1010 